\title{
HYDROXYL RADICAL INDUCED ULTRA-WEAK PHOTON EMISSION FROM TYROSINE SOLUTIONS
}

This paper deals with ultra-weak photon emission (UPE) from aqueous tyrosine solutions induced by hydroxyl radical. The physical nature of this specific phenomenon is presented and the proposed mechanism of its origin during oxidation of tyrosine is described. The experimental part of the work is focused on the measurements of intensity of ultra-weak photon emission from different solutions of tyrosine. The emission from solutions with and without presence of hydroxyl radical is analyzed. The results confirm the assumption that the highest intensity is detected from the solution of tyrosine, hydrogen peroxide and ferrous sulphate heptahydrate, likely due the Fenton reaction originated hydroxyl radical, which causes oxidation of tyrosine, formation of high-energy intermediates and electron excited species. Since hydroxyl radical induced ultra-weak photon emission from tyrosine has not been analyzed before in the available literature, our results could be the beneficial contribution to this field of research.

Keywords: Ultra-weak photon emission, reactive oxygen species, tyrosine, hydroxyl radical.

\section{Introduction}

The emission of light, originated from chemical processes in organisms is an externally detectable manifestation of the metabolic activity of living cells. One of the most commonly used terms for this kind of light emission is ultra-weak photon emission (UPE). Due to its non-invasive, low-operation-cost and label-free application, it can be used in many fields of biomedicine, such as dermatology, neuroscience or oncology [1].

Ultra-weak photon emission originates during relaxation of electronically excited species, which are generated chemically by oxidative reactions of reactive oxygen species (ROS) with biomolecules. The formation of ROS is caused by physical, chemical and biological stimuli [2]. ROS can be distinguished in two main groups - radical ROS and non-radical ROS. Molecules of radical ROS have one or more unpaired electrons, and therefore they are highly reactive. Non-radical ROS have no unpaired electron, but they still exhibit rather high reactivity. ROS oxidize biomolecules and these reactions can further lead to the formation of intermediate chemical species which decay to form electronically excited species. The transition of these species to the ground state is accompanied by the photon emission in near UVA ( $350-400$ $\mathrm{nm})$, visible $(400-750 \mathrm{~nm})$ and near IR $(750-1300 \mathrm{~nm})$ regions of the electromagnetic spectrum [1].
ROS have an important role in biochemical reactions in living cells. They have a great significance in intracellular signaling [3] and intercellular communication [4]. On the other hand, there is also an extensive evidence for harmful effects of excessive ROS levels in organisms [5]. It is known that reactions of radical species can be affected by magnetic field. There are many scientific works dealing with magnetic field effects on free radical reactions and radical pair mechanism [6 - 11]. Besides these works, numerous experimental works have shown that magnetic field can affect metabolic processes of cells [12 - 14]. Therefore it could be beneficial to find a connection between the mechanism of magnetic field effects on radical reactions and the evaluation of its influence on cell metabolism by the UPE detection.

UPE can be considered as a product of biochemical processes in living organisms, which involves oxidation of bio-molecules [15].The subject matter of this paper is focused on ultra-weak photon emission derived from oxidation of amino-acid tyrosine mediated by hydroxyl radical. Tyrosine is aromatic nonessential amino-acid formed in organism from phenylalanine. Tyrosine is a building block for several important neurotransmitters, such as epinephrine or dopamine. It also underlies production of melanin, the pigment responsible for hair and skin color [16]. In this paper, it is shown that chemically produced hydroxyl radical is responsible for oxidation of tyrosine; and there are presented

\footnotetext{
* ${ }^{1}$ Martin Bereta, ${ }^{2}$ Michal Cifra, ${ }^{2}$ Katerina Cervinkova, ${ }^{1}$ Ladislav Janousek, ${ }^{3}$ Jan Barabas

${ }^{1}$ Department of Electromagnetic and Biomedical Engineering, Faculty of Electrical Engineering, University of Zilina, Slovakia

${ }^{2}$ Institute of Photonics and Electronics, Czech Academy of Sciences, Prague, Czech Republic

${ }^{3}$ University Science Park of the University of Zilina, Slovakia

E-mail: martin.bereta@fel.uniza.sk
} 
experimental results of detection of ultra-weak photon emission from tyrosine aqueous solution.

\section{Mechanism of ultra-weak photon emission from tyrosine}

It is known that the oxidation of amino-acids and proteins is also associated with ultra-weak photon emission [17 and 18]. Tyrosine is one of the residues which are most prone to oxidation by hydroxyl radical (i.e. tyrosine has a relatively low reduction potential [19]) in proteins and its oxidation can be monitored by detection of ultra-weak photon emission. Oxidation of tyrosine mediated by hydroxyl radical involves a one-electron oxidation to form the tyrosyl radicals. It is known that hydroxyl radical originates from hydrogen peroxide by Fenton reaction:

$\mathrm{Fe}^{2+}+\mathrm{H}_{2} \mathrm{O}_{2} \rightarrow \mathrm{Fe}^{3+}+{ }^{\cdot} \mathrm{OH}+\mathrm{OH}^{-}$.

The interaction of hydroxyl radical with tyrosine initiates oxidative reactions. Tyrosine molecule contains an aromatic ring. The computational study of Mujika et al. [20] analyzed oxidation of some aromatic amino-acids including tyrosine. They considered two reaction pathways by the oxidation process: addition of hydroxyl radical to the aromatic ring and hydrogen abstraction from all of the possible side-chain atoms. The energy barriers for hydrogen abstraction from aromatic ring are slightly larger and thermodynamically less favorable than for addition processes. However, hydrogen abstraction from the beta-carbon $\left(\mathrm{C}_{7}\right)$ or from the $\mathrm{O}_{8}$ atom show similar energy barriers as the addition processes and both these abstraction processes are thermodynamically favored. Hydrogen abstraction from the $\mathrm{O}_{8}$ atom (Fig. 1) would be sterically favorable compared to from the beta-carbon.
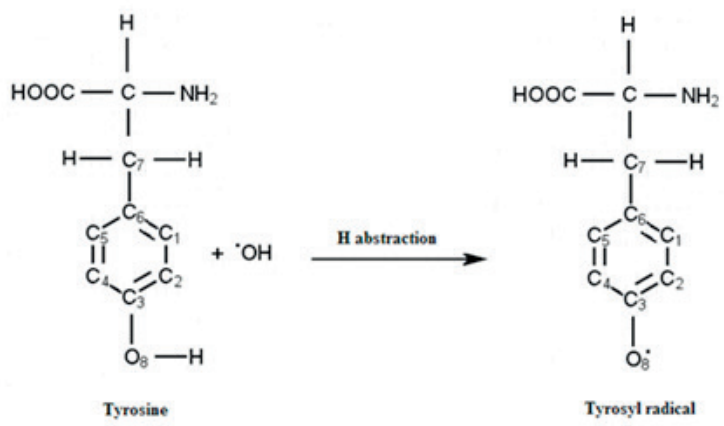

Fig. 1 The most energetically and thermodynamically favored hydroxyl radical attack on tyrosine molecule

Summarizing the Mujika computational results it seems that hydrogen abstraction from the $\mathrm{O}_{8}$ atom is the most favorable hydroxyl radical attack on tyrosine molecule. Next favored attack is the $\mathrm{H}$ abstraction from beta-carbon and then the addition of $\mathrm{OH}$ radicals to the aromatic ring $\left(\mathrm{C}_{1}-\mathrm{C}_{6}\right)$. The least favorable reactions are $\mathrm{H}$ abstraction from $\mathrm{C}_{1}, \mathrm{C}_{2}, \mathrm{C}_{4}$ and $\mathrm{C}_{5}$ atoms. Following the aim of the paper to try to propose possible mechanism leading to the formation of electronically excited species, hydrogen abstraction from $\mathrm{O}_{8}$ atom is one of the expected reactions that could initiate this process. It is generally considered that reaction pathway resulting to the excited species origin involves the formation of intermediates tetroxide (ROOOOR) or dioxetane (ROOR) [ 1 and 2]. In the case of tyrosine oxidation by hydroxyl radical, tetroxide could be formed by the recombination of two tyrosyl radicals (on $\mathrm{O}_{8}$ ) and addition of molecular oxygen. Since the formation of dioxetane requires the cyclization of peroxyl radicals ( $\mathrm{ROO} \bullet$ ) molecules or cycloaddition of singlet oxygen $\left({ }^{1} \mathrm{O}_{2}\right)$ [2], its origin in this oxidation process (hydrogen abstraction from $\mathrm{O}_{8}$ ) seems to be lowly probable, though not absolutely excluded. It is expected that the decomposition of tetroxide would be then followed by the formation of excited triplet carbonyl or singlet oxygen and ground state carbonyl [1]. However, we are not able to distinguish from the current data which pathway is dominant or if there is an additional unknown reaction pathway taking place.

The transition of the excited triplet carbonyl to the ground state can be accompanied by ultra-weak photon emission. Besides this, energy of the excited carbonyl could be transferred to the molecular oxygen and lead to the formation of singlet oxygen that subsequently can also emit a photon. Dimol emission of singlet oxygen lies in the visible region of the spectra. The reaction pathway of the dioxetane decomposition would be identical besides the possibility of the direct singlet oxygen formation [2]. The proposed principal mechanism of the origin of UPE during tyrosine oxidation by hydroxyl radical, following the results of Mujika computational study is shown in Fig. 2.

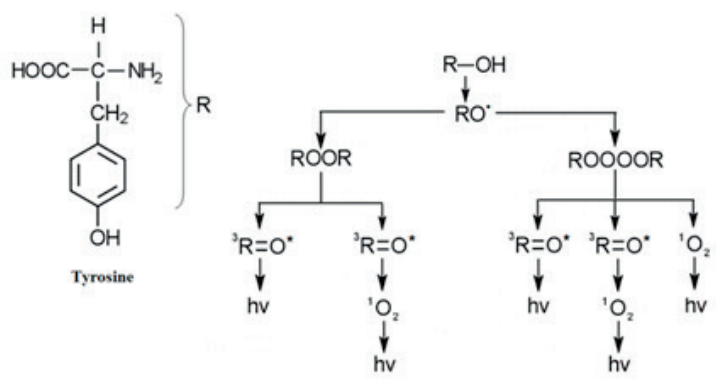

Fig. 2 Proposed mechanism of the origin of photon emission from tyrosine involves radical reactions, formation of intermediates and electron excited species (adopted from [2])

\section{Materials and methods}

The experimental part of the paper is focused on the detection of ultra-weak photon emission from different solutions of aminoacid tyrosine. In the experiments these compounds are used: 
tyrosine (P-Lab, CZ, purity $99 \%$ ), $30 \%$ hydrogen peroxide (Penta, CZ, p.a.), ferrous sulphate heptahydrate (Penta, CZ, purity $99 \%)$. Four different solutions of tyrosine are prepared. The first solution is composed of tyrosine with molecular concentration $0.38 \mathrm{mM}$. The second solution contains tyrosine $(0.38 \mathrm{mM})$ and hydrogen peroxide $(166.7 \mathrm{mM})$. The third solution consists of tyrosine $(0.38 \mathrm{mM})$ and ferrous sulphate heptahydrate, where the concentration of iron is $0.27 \mathrm{mM}$. The fourth solution is composed of tyrosine $(0.38 \mathrm{mM})$ with presence of hydrogen peroxide $(166.7 \mathrm{mM})$ and ferrous sulphate heptahydrate $\left(\mathrm{FeSO}_{4} \cdot 7 \mathrm{H}_{2} \mathrm{O}\right)$, with the same concentration of iron as in previous case. All the mentioned concentrations of individual components represent their final concentrations in the measured solution. Purified water and solution of hydrogen peroxide $(166.7 \mathrm{mM})$ and ferrous sulphate heptahydrate $(0.27 \mathrm{mM}$ of iron $)$ are used as control samples.

Photomultiplier tube H7360-01, selected type (Hamamatsu Photonics K. K.) with a spectral sensitivity in the range $300-650 \mathrm{~nm}$ is used to detect photon emission. Typical dark count (noise) of the photomultiplier (PMT) is 15 counts per second. Measurement of the samples takes place in a lighttight chamber (standard black box, Institute of Photonics and Electronics, CZ) specially designed for the purposes of UPE measurements. The photomultiplier is mounted on the top of the chamber viewing a sample inside the chamber. Measurement equipment can be seen in Fig. 3 .

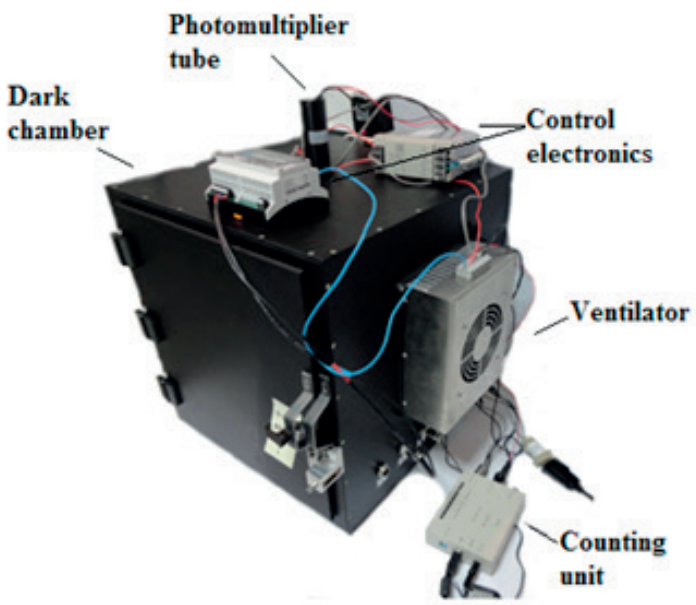

Fig. 3 Measurement equipment consists of the dark chamber, photomultiplier tube, control electronics, ventilator and counting unit

The temperature in laboratory is about $21^{\circ} \mathrm{C}$ in all the measurements. The duration of every measurement is one hour. The volume of measured samples is $9 \mathrm{ml}$ in all cases. Every experiment is performed in triplicate.

\section{Results and discussion}

The results of ultra-weak photon emission measurement are presented in the following figures. Figure 4 shows results of the intensity of ultra-weak photon emission from water, solution of tyrosine, solution of tyrosine with presence of hydrogen peroxide and with presence of ferrous ions and solution of hydrogen peroxide and ferrous sulphate. The results represent the mean intensity of UPE, calculated from mean values of three repetitions of every experiment. The error bars constitute the standard deviation of the mean values. It can be seen that the intensity of UPE is very similar for both solutions water and tyrosine and not distinguishable from the PMT dark count. No detectable photon emission arises in these cases. In the case of the solution of tyrosine and hydrogen peroxide $\left(\mathrm{H}_{2} \mathrm{O}_{2}\right)$, an increase of the UPE intensity can be seen. Although hydroxyl radicals are not formed in the solution, some molecules of tyrosine are probably oxidized by molecular oxygen or by products of the autooxidation of hydrogen peroxide. Electronically excited species are then formed from oxidation products of these reactions. An increase of intensity of UPE is observed also from control solution of hydrogen peroxide and ferrous sulphate. Hydroxyl radical originated by the Fenton reaction could likely oxidize some molecules of air in chamber, which could lead to the formation of excited species and photon emission.

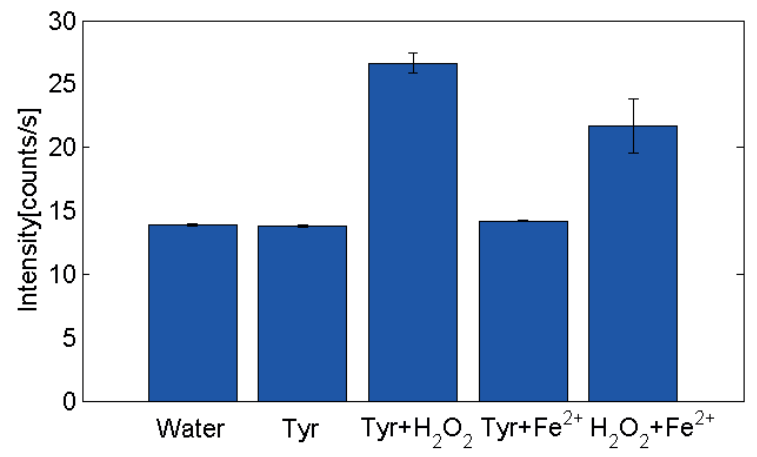

Fig. 4 Averaged intensity of ultra-weak photon emission from different solutions of tyrosine and control solutions

After the addition of ferrous sulphate to the solution of tyrosine and hydrogen peroxide and following Fenton reaction, the notable increase of photon emission is detected. The results of UPE for three repetitions of the experiment are shown in Fig. 5. The first peak in the UPE kinetics is likely caused by a fast oxidation of tyrosine molecules by hydroxyl radical. Total final shape of UPE intensity is likely due to the multiple reaction pathways as described in Fig. 2.

The observed results confirm the key role of hydroxyl radical in oxidation processes leading to the photon emission from tyrosine. Although any exact mechanism of UPE from tyrosine is not actually known, the presented reaction scheme (Fig. 2) could 
be expected as one of the pathway of origin of light emission from tyrosine.

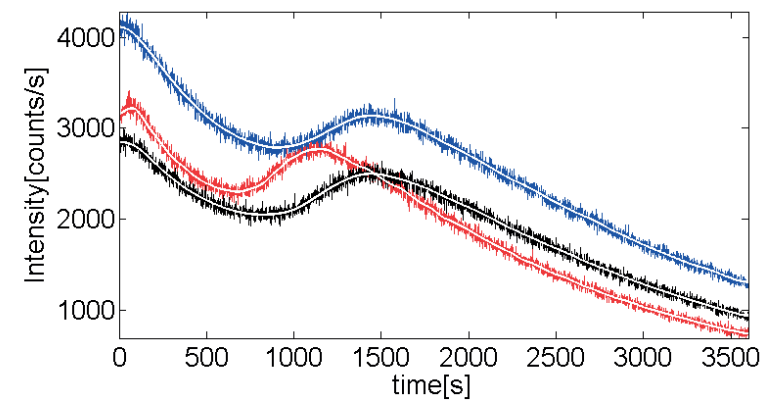

Fig. 5 Ultra-weak photon emission solution of tyrosine, hydrogen peroxide and ferrous sulphate manifests high intensity compared to control samples.

\section{Conclusion}

The detection of ultra-weak photon emission from different solutions of tyrosine was presented in this paper. The results confirmed the assumption that the highest intensity of UPE is detected from solution of tyrosine with the presence of hydrogen peroxide and ferrous sulphate, likely due to the hydroxyl radical mediated oxidation of tyrosine. The kinetics of UPE intensity from tyrosine without presence of hydrogen peroxide and from purified water was not distinguishable from dark count (noise) of photomultiplier. As it was noted above, UPE can be detected also from living cells and can be likely affected by magnetic field. Possible differences in kinetics of UPE from cells exposed to magnetic field and from unexposed cells could indicate the changes in biochemical reactions during cell metabolism. Our next research will be focused on evaluation of magnetic field effects on cells using UPE detection.

\section{Acknowledgement}

The research presented in the paper has been financially supported by the Czech Science Foundation, grant no. GP1329294S and by the following project: University Science Park of the University of Zilina (ITMS: 26220220184) supported by the Research\&Development Operational Program funded by the European Regional Development Fund. The research was carried out during Short Term Scientific Mission 21626 of the COST Action BM1309. Jiri Prusa is acknowledged for helpful comments and Michaela Poplova for help with results processing.

\section{References}

[1] CIFRA, M., POSPISIL, P.: Ultra-weak Photon Emission from Biological Samples: Definition, Mechanisms, Properties, Detection and Applications. J. of Photochemistry and Photobiology B: Biology, vol. 139, pp. 2-10, 2014.

[2] POSPISIL, P., et al.: Role of Reactive Oxygen Species in Ultra-weak Photon Emission in Biological Systems. J. of Photochemistry and Photobiology B: Biology, vol. 139, pp. 11-23, 2014.

[3] LANDER, H. M.: An Essential Role for Free Radicals and Derived Species in Signal Transduction. The FASEB J., vol. 11, No. 2, pp. 118-124, 1997.

[4] THANNICKAL, V. J. FANBURG, B. L.: Reactive Oxygen Species in Cell Signaling. American J. of Physiology, vol. 279, no. 6, pp. 1005-1028, 2000.

[5] WARIS, G., ASHAN, H.: Reactive Oxygen Species: Role in the Development of Cancer and Various Chronic Conditions. J. of Carcinogenesis, vol. 5, No. 14, 2006.

[6] ENGSTROM, S.: Magnetic Field Effects on Free Radical Reactions in Biology. Bioengineering and Biophysical Aspects of Electromagnetic Fields, $3^{\text {rd }}$ ed., CRC Press: Boca Raton _ Fla, 2007. ISBN: 978-0-8493-9539-0

[7] BARNES, F.S. GREENEBAUM, B.: The Effects of Weak Magnetic Fields on Radical Pairs. Bioelectromagnetics, vol. 36, pp. 45-54, 2015.

[8] WOODWARD, J.R.: Radical Pairs in Solution. Progress in Reaction Kinetics and Mechanism, vol. 27, pp. 165-207, 2002.

[9] TIMMEL, C. R. et al.: Effects of Weak Magnetic Fields on Free Radical Recombination Reactions. Molecular Physics, vol. 95, No. 1 , pp. $71-89,1998$.

[10] OKANO, H.: Effects of Static Magnetic Fields in Biology: Role of Free Radicals. Frontiers in Bioscience, vol. 13, pp. 6106-6125, 2008.

[11] STEINER, U.E., ULRICH, T.: Magnetic Field Effects in Chemical Kinetics and Related Phenomena. Chemical Reviews, vol. 89, pp. 51-147, 1989.

[12] BARABAS, J., RADIL, R.: Evidence of S. Cerevisiae Proliferation Rate via Exogenous Low Frequency Electromagnetic Field. Information Technologies in Biomedicine, vol. 7339, pp. 295-303, 2012. 
[13] AKAN, Z., et al.: Extremely Low-frequency Electromagnetic Fields Affect the Immune Response of Monocyte-derived Macrophages to Pathogens. Bioelectromagnetics, vol. 31, pp.603-612, 2010.

[14] AKDAG, M.Z., et al: Alteration of Nitric Oxide Production in Rats Exposed to a Prolonged, Extremely Low-frequency Magnetic Field. Electromagnetic Biology and Medicine, vol. 26, pp. 99-106, 2007.

[15] KHABIRI, F., et al:: Non-invasive Monitoring of Oxidative Skin Stress by Ultraweak Photon Emission (UPE)-measurement. I: Mechanisms of UPE of Biological Materials. Skin Research and Technology, vol. 14, pp. 103-111, 2008.

[16] Tyrosine. [online][cit. 2015-03-10] 〈http://umm.edu/health/medical/altmed/supplement/tyrosine〉

[17] BARNARD, M. L., et al.: Protein and Amino Acid Oxidation is Associated with Increased Chemiluminescence. Archives of Biochemistry and Biophysics, vol. 300, No. 2, pp. 651-656, 1993.

[18] FEDOROVA, G. F. et al.: Peroxy-radical-mediated Chemiluminescence: Mechanistic Diversity and Fundamentals for Antioxidant Assay. Arkivoc, pp. 163-215, 2007.

[19] MILligAN, J. R., et al. Repair of Oxidative DNA Damage by Amino Acids. Nucleic Acids Research, vol. 31, pp.6258-6263, 2003.

[20] MUJIKA, J. I., et al.: Computational Study on the Attack of 'OH Radicals on Aromatic Amino-acids. Chemistry-A European J., vol. 19, pp. 6862-73, 2013. 